\title{
Surface-modified polyolefin microfluidic devices for liquid handling
}

\author{
Rongsheng $\operatorname{Lin}^{1,3}$ and Mark A Burns ${ }^{1,2}$ \\ ${ }^{1}$ Department of Chemical Engineering, The University of Michigan, Ann Arbor, \\ MI 48109-2136, USA \\ ${ }^{2}$ Department of Biomedical Engineering, The University of Michigan, Ann Arbor, \\ MI 48109-2136, USA \\ ${ }^{3}$ Department of Electrical Engineering, The University of Michigan, Ann Arbor, \\ MI 48109-2136, USA \\ E-mail: maburns@umich.edu
}

Received 3 August 2005, in final form 23 September 2005

Published 10 October 2005

Online at stacks.iop.org/JMM/15/2156

\begin{abstract}
Polymer-based microfluidic devices offer an attractive platform for single-use disposable applications due to their low cost, ease of fabrication and good biocompatibility. In this work, we investigated liquid handling in surface modified polyolefin microfluidic devices. The modification of the surface was accomplished using ultraviolet light, and the contact angle was reduced from $88^{\circ}$ to $45^{\circ}$. This type of treatment is easy to implement and could be beneficial for liquid handling in microchannel networks.

Capillary-driven flow, contact angle hysteresis and pulsed pumping were demonstrated in these plastic devices. This surface treatment also facilitates rapid gel loading for separation since viscous sieving media can be injected solely by capillary force. Nucleic acid separation was demonstrated in the gel-loaded devices.
\end{abstract}

(Some figures in this article are in colour only in the electronic version)

\section{Introduction}

The development of microfluidic systems for many routine tasks has been a significant area of research in chemistry, biology and medicine in recent years due to the reduction in analysis time and reagent volumes resulting from the miniaturized format. Great strides have been made in applying microfluidics for genomics (DNA genotyping or sequencing) [1-3], proteomics (protein identification) [4-7], and clinical diagnostics (virus or pathogen detection) $[8,9]$. However, substrate materials have to be chosen carefully in terms of cost, biocompatibility, mechanical, chemical and optical properties, ease of fabrication and other properties. Much of the initial work on miniaturized chemical analysis systems has centered on the use of glass or silicon substrates along with the standard lithographic fabrication technology [10]. Compared to glass and silicon, polymers are attractive materials ideally suitable for single-use disposable devices since they offer low cost, ease of fabrication and good biocompatibility.

There have been increasing efforts to use polymeric materials for chip-based devices. Several extensive reviews have discussed fabrication techniques of polymer microfluidic devices including casting, laser ablation, imprinting, hot embossing and injection molding along with their applications for genetic analysis [11-13]. Various polymeric materials, including poly(dimethylsiloxane) (PDMS), poly(methyl methacrylate) (PMMA) and polycarbonate (PC) have been employed in the fabrication of microfluidic devices.

Most of the commercially available polymers for microfluidic applications are hydrophobic. These materials include polycarbonate (PC), poly(methyl methacrylate) (PMMA), polydimethysiloxane (PDMS) and copolymer of 2norbornene ethylene ('cyclic olefin copolymer', COC). The hydrophobic nature could be problematic for liquid handling in microfluidic devices. Unlike loading samples in hydrophilic channels using capillary force, external pumping is necessary to wet hydrophobic channels. As a result of hydrophobic interactions, the surfaces may capture specific compounds from the solution passing through the channels, changing their concentration in the solution and affecting the reliability of quantitative assays. Appropriate functionalization of the surface of the polymer microchannels would enable control 
Surface modified polyolefin microfluidic devices for liquid handling

of the flow and adsorption processes and greatly improve the reliability of these polymer-based microchips.

The current literature presents numerous examples of surface modifications on a broad range of polymers. Most of these procedures are not suitable for microfluidic chips since they rarely allow selective patterning on the device. This requirement favors processes triggered by UV light [14-18]. Among the most commonly used polymer materials, cyclic olefin copolymer has an excellent optical clarity close to glass and PDMS in the ultraviolet light range $(>80 \%$ transmission at $320 \mathrm{~nm}$ wavelength) [19] and low background fluorescence [20]. Because of its good combination of optical clarity, mechanical strength and low cost, this material has been used to fabricate microfluidc devices for clinical diagnostics [21, 22].

We investigated fluidic handling in surface-modified polyolefin microchannel networks. These devices were fabricated using a hot embossing method, and the surface was modified using a UV-mediated grafting technique. We investigated the imbibing flow driven by capillary force, pulsed drop motion, contact angle hysteresis and loading of separation media. Application of these devices for nucleic acid separation was demonstrated in the assembled polymer devices.

\section{Materials and methods}

\section{Device fabrication}

Mold fabrication. The fabrication process for the polymeric microfluidic chip is depicted in figure 1 and is a modification of a published procedure [23, 24]. The Shipley AZ9620 photoresist (Microchem, Newton, MA) was spin-coated (2000 rpm for $30 \mathrm{~s}$ ) on the silicon wafer and softbaked at $90{ }^{\circ} \mathrm{C}$ for $2.5 \mathrm{~min}$. A contact aligner (Cannon PLA 501FA, $405 \mathrm{~nm})$ was used to expose the photoresist to UV light (405 $\mathrm{nm}, 10 \mathrm{~mW} \mathrm{~cm} \mathrm{c}^{-2}$ ) for $50 \mathrm{~s}$, and the resulting wafer was developed using an $\mathrm{AZ} 400 \mathrm{~K}$ developer $\left(\mathrm{AZ400K}: \mathrm{H}_{2} \mathrm{O}=\right.$ 1:3) for $30 \mathrm{~s}$. After a hard bake at $110^{\circ} \mathrm{C}$ for $30 \mathrm{~min}$, the wafer was etched in a buffered HF solution for 4 min to remove the top silicon dioxide layer (etch rate approx. $100 \mathrm{~nm} \mathrm{~min}^{-1}$ ) and then was placed in a silicon deep reactive ion etcher to etch away the silicon to the desired depth of 50-100 $\mu \mathrm{m}$. The remaining photoresist was then removed using acetone.

Hot embossing. The COC polymer (Zenoex 480, $T_{\mathrm{g}}=$ $138{ }^{\circ} \mathrm{C}$, Zeon Chemicals, Louisville, KY) was cleaned with acetone for $2 \mathrm{~min}$ in an ultrasonic bath and cut into the desired size $(4 \mathrm{~cm} \times 4 \mathrm{~cm})$. The chip was exposed to the silicon master with heat $\left(154{ }^{\circ} \mathrm{C}\right)$ and pressure $(250 \mathrm{psi})$ from both top and bottom plates of the hot-press machine (Hot Press 4122, Canrer Inc., Wabash, IN) for 10-15 min. The chip and the silicon master then cooled down naturally to release the stress. Care had to be taken to completely detach the chip and the master to avoid breaking the silicon mold. The depth of the channel was then measured using Alpha-step 500 surface profiler (KLA\&Tencor). Access holes $(300 \mu \mathrm{m}-2 \mathrm{~mm})$ were drilled in the desired location using a drilling machine. The two plastic pieces were bonded by application of pressure (695 psi) and heat $\left(123{ }^{\circ} \mathrm{C}\right)$ for $10-15$ min using the same hot press machine.

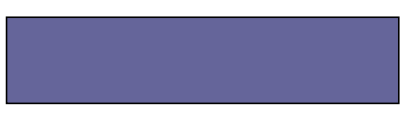

Substrate (silicon)

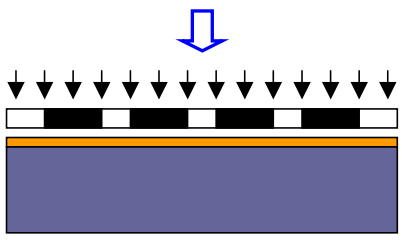

Pattern photoresist
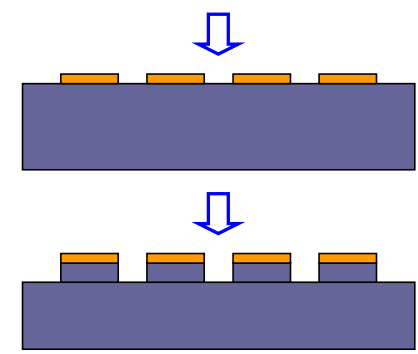

Etch substrate (silicon: DRIE)

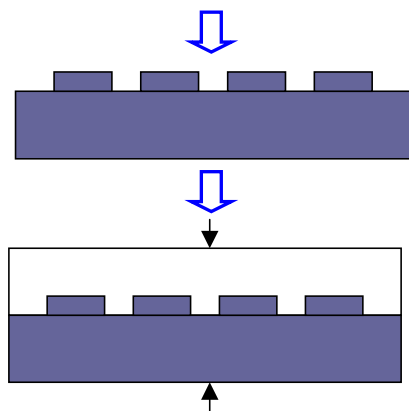

Remove photoresist

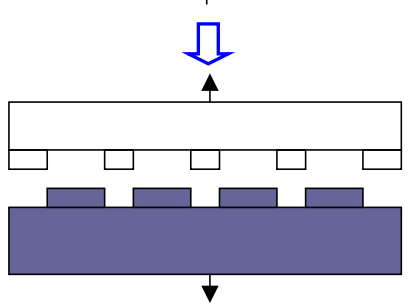

Hot emboss plastic channels

Release the mold

Figure 1. Process flow to fabricate a silicon mold and hot-embossed plastic devices.

In addition to the wafer-level thermal bonding, individual plastic pieces were bonded using an optical adhesive (SK-9 Lens Bond, Summers Laboratories, Fort Washington, PA). The plastic surfaces were typically treated to become hydrophilic using the photografting procedure described in the next section. The glue was applied sparingly to the edge of the device and allowed to wick into the gap. The bond was cured under an ultraviolet light source $(365 \mathrm{~nm})$ for $30 \mathrm{~min}$. The cross-section SEM pictures were prepared by a scanning electron microscope (Philips XL 30, Philips Electron Optics).

PDMS device fabrication. PDMS devices were also fabricated using the same silicon mold. PDMS base and curing agent (Sylgard 184; Dow Corning) were mixed at 1:10 ratio, degassed in a vacuum chamber until no bubbles were observed. The mixture was then poured onto the silicon mold and cured at $70{ }^{\circ} \mathrm{C}$ for $3 \mathrm{~h}$. To bond two pieces of PDMS, the substrates were cleaned with IPA and oxidized in a plasma etcher (Reactive Ion Etcher 2000, South Bay Technologies) for $1 \mathrm{~min}$. The two pieces were then immediately pressed gently into contact and placed in an oven at $90{ }^{\circ} \mathrm{C}$ for $10 \mathrm{~min}$ to ensure the bonding. 


\section{Grafting procedure}

Benzophenone was dissolved in ethanol to make the final concentration of $3.3 \%(\mathrm{w} / \mathrm{v}) .40 \%(\mathrm{w} / \mathrm{v})$ acrylamide solution was made by dissolving acrylamide into DI water. Pluronic F68 was dissolved into DI water to make a $3.3 \%(\mathrm{w} / \mathrm{v})$ concentration. The final grafting solution was made by mixing the $40 \%$ acrylamide, $3.3 \%$ benzophenone, 3.3\% Pluronic F68 and DI water in a ratio of 7.5:1:1:0.5. The final concentrations of acrylamide, benzophenone and Pluronic F68 are 30\%, $0.33 \%$ and $0.33 \%(\mathrm{w} / \mathrm{v})$, respectively.

A small drop of grafting solution was placed on the COC bottom plate with another COC plate as cover. The sandwich was illuminated under a UV light source (Spectroline microprocessor-controlled UV crosslinker XL-1000, $365 \mathrm{~nm}$, $40 \mathrm{~W}$, Fisher Scientific, USA) at a distance of 3 to $4 \mathrm{~cm}$ for 10-20 min until the color of the solution was changed to white. The two plates were detached, rinsed by DI water for $10 \mathrm{~min}$ and soaked in the water bath for $12 \mathrm{~h}$ before use. The similar procedure was performed to modify the surface in the microchannels.

\section{Contact angle measurement}

A water drop $(\sim 0.5 \mu \mathrm{L})$ was gently placed on the experimental surface, and a photograph of the side profile of the liquid drop was taken using a CCD camera with an adjustable optical focus lens. The radius of the spherical cap (gravity is negligible at this size) was measured by processing the side-view image to extract the free surface of the spherical cap and fitting it with a circle using ImageJ software (National Institutes of Health, Bethesda, MD (http://rsb.info.nih.gov/ij)). The contact angle was calculated from the radius $R$ of the fitting circle and the height $h$ of the droplet using $\theta=\cos ^{-1}\left(\frac{R-h}{R}\right)$.

\section{Fluidic test}

Lab air, controlled by a pressure regulator (Matheson Gas Products Inc., Irving, TX, Model Number 3701), was connected via a solenoid valve (Numatics Inc., Model Number LS02L6H00B) to the microfluidic chip. Pulsed pumping was done by the opening and closing of the solenoid valve, controlled through a combination of a dc power supply $(+12 \mathrm{~V} \mathrm{dc}$, Electro Industries, Model Digi 35A) and a relay board (National Instruments, TX, Model ER-16). The relay board is controlled through a program written in LabVIEW (National Instruments, TX) and a Digital I/O card (PCIDIO-96). The pulse number and duration were input in LabVIEW. The pressure and vacuum was measured using a digital pressure meter.

\section{Chemicals}

The polyethylene oxide (PEO, MW 400000-4000000) solutions were prepared by dissolving the appropriate amount of polymer powder in $1 \mathrm{X}$ TBE separation buffer solution (89 mM Tris, $89 \mathrm{mM}$ borate, $2 \mathrm{mM}$ EDTA, $\mathrm{pH}$ 8.3). The hydroxypropylmethyl cellulose (HPMC) solution was prepared by dissolving the appropriate amount of the stock solution $(2 \%)$ and glucose powder $(8 \%)$ in $1 \mathrm{X}$ TBE buffer. After being gently stirred overnight, these solutions were then degassed and left undisturbed for $12 \mathrm{~h}$. The viscosity was measured on the AR1000 rheometer (TA instruments rheology division ST-B). A $2^{\circ}$ cone and $6 \mathrm{~cm}$ plate was used.

\section{Separation}

$100 \mathrm{bp}$ dsDNA standard ladder was fluorescently labeled with YOYO-1 dye at a ratio of 5:1. The migrating bands were detected using an Olympus SZX-12 fluorescence stereoscope with a mercury arc illumination source and a CCD camera (Hamamatsu C2400-08 SIT, Hamamatsu Corporation, USA, Bridgewater, NJ) for imaging. The camera output was recorded and digitized. Intensity profiles corresponding to the migrating bands were obtained by extracting the variation in fluorescence intensity with time at a fixed location in the gel using Transform 2D image analysis software (Research Systems, Inc., Boulder, CO).

\section{Results and discussions}

\section{Surface modification}

The fabrication process to generate silicon master and polyolefin plastic chips is shown in figure 1 . The choice of silicon as a master material is due to the maturity of silicon surface and bulk micromaching techniques. In addition to planar structures, three-dimensional topology can also be readily realized giving the ability of fabricating threedimensional silicon molds. Figure 2 shows the silicon mold and the devices fabricated using this silicon mold. The anisotropic nature of dry etching produces an excellent crosssection profile of the resulting microchannels.

The polymer surface can be modified through ultraviolet illumination, switching the surface from hydrophobic to hydrophilic. The static contact angle of water on a native COC surface is $88^{\circ} \pm 2.0^{\circ}$, whereas the treated COC surface has a lower contact angle around $45^{\circ} \pm 4.0^{\circ}$. This method offers a relatively constant hydrophilic surface after grafting. We monitored the contact angle over the period of one week, and no obvious aging effects were observed (figure 3 ).

An advantage of other techniques such as oxygen plasma treatment [25] is that they allow the hydrophilicity of the surface to be adjusted by varying the reaction conditions (e.g. gas for plasma, power, duration) [22]. In our studies, we saw no difference in surface hydrophilicity as a function of UV illumination although our range was rather limited (10-20 min of exposure, constant source). Recent results show that the contact angle can indeed be tuned by varying UV illumination time [26]. Further optimization of UV conditions may extend the UV grafting techniques to a variety of applications.

\section{Fluidic handling}

The surface treatment facilitates the loading of the liquid through capillary force. Figure 4 shows the meniscus position as a function of time after a drop of DI water $(\sim 5 \mu \mathrm{L})$ is placed on the inlet of the microchannel. A comparison between capillary-driven flow in the untreated and treated COC devices, glass chips (natively hydrophilic) and oxygen plasma treated PDMS microchannels shows similar fluidic behaviors. Due 
Surface modified polyolefin microfluidic devices for liquid handling

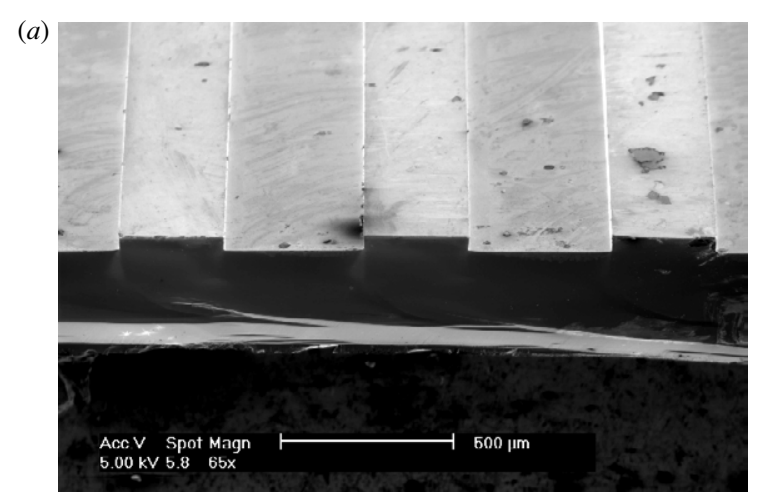

(b)

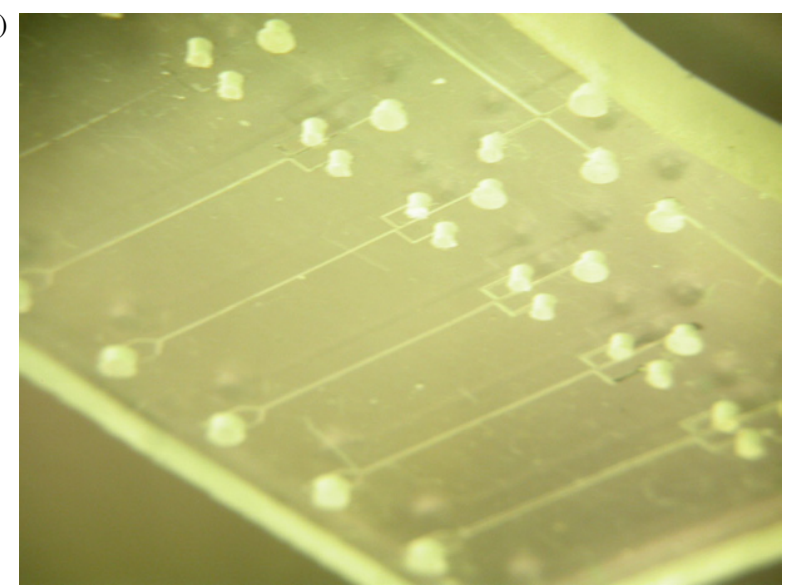

(c)

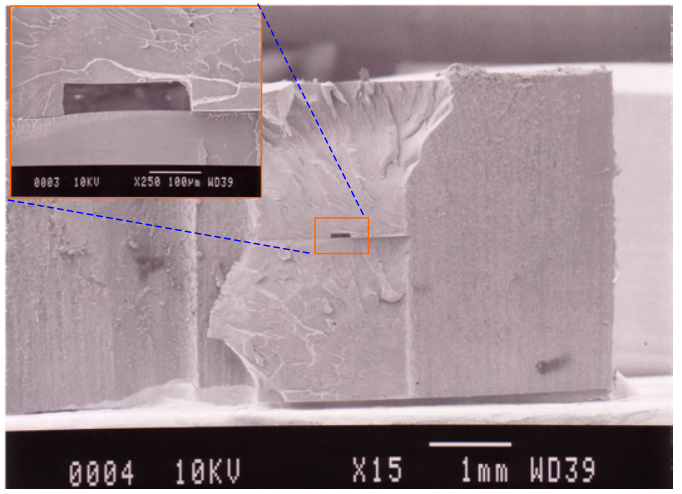

Figure 2. (a) Scanning electron micrograph of the microfabricated silicon mold. (b) Pictures of hot-embossed plastic devices.

(c) Scanning electron micrograph of the rectangular cross-section of the plastic microchannel.

to the aging effect, the hydrophilicity of the PDMS surface dramatically decreases after remaining in air for as little as $11 \mathrm{~h}$. After 6 days, the water cannot be loaded into the channel by capillary force, which is similar to native COC microchannels. Note that keeping the oxidized PDMS in contact with water can maintain a stable hydrophilic surface for an extended period of time [27].

In addition to capillary-driven flow, drop movement and positioning are also of significance for droplet-based microfluidic analysis devices [10, 28]. Instead of constant pneumatic actuation, a pulsed pressure system for moving and positioning drops in microchannels results in a more flexible and controllable fashion. Short (10-100 ms) pulses of air at moderate pressures (1-10 psi) were applied to move the drop

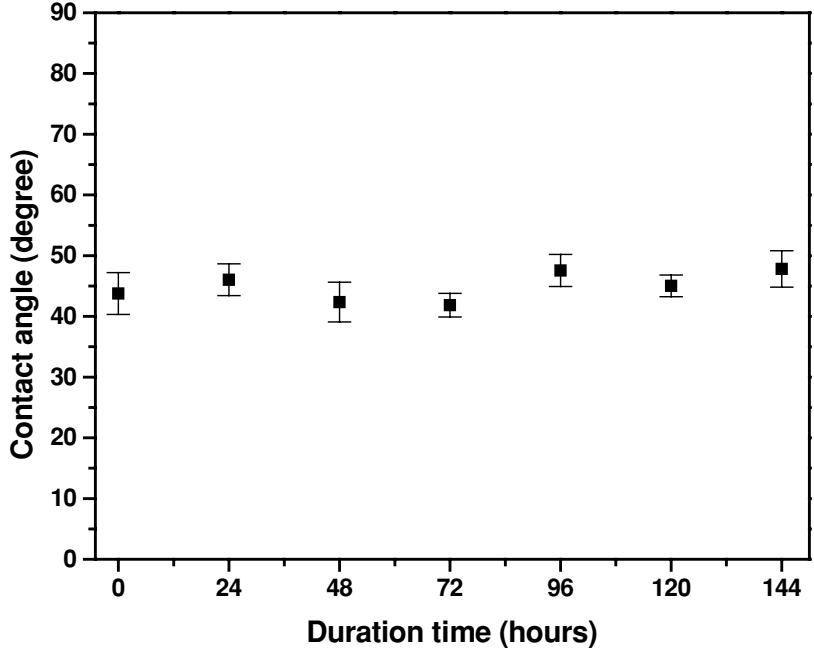

Figure 3. Contact angle of the photo-grafted COC surface as a function of duration time in the air at room temperature.
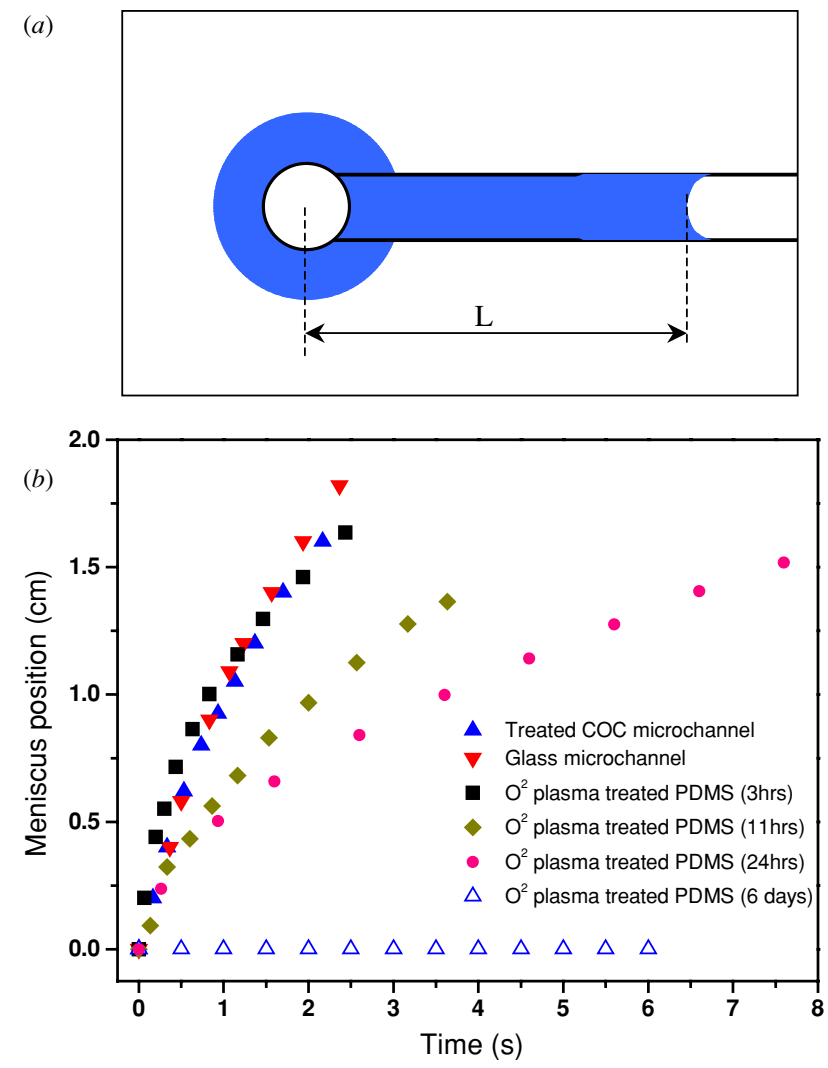

Figure 4. (a) Schematic drawing showing capillary-driven flow (top view). (b) The meniscus position $(L)$ as a function of time inside the microchannel. $(\boldsymbol{\Delta})$ denotes treated COC $(300 \mu \mathrm{m} \times$ $60 \mu \mathrm{m})$. ( $\mathbf{\nabla})$ denotes glass $(500 \mu \mathrm{m} \times 45 \mu \mathrm{m})$. ( $\times),(\bullet),(\bullet),(\Delta)$ denotes PDMS exposed in the air for $3 \mathrm{~h}, 11 \mathrm{~h}, 24 \mathrm{~h}$ and 6 days after oxygen plasma treatment respectively. The channel dimension is $300 \mu \mathrm{m} \times 60 \mu \mathrm{m}$.

a fixed distance per pulse (figure 5(a)). The total distance moved varies linearly with the number of pulses (figure $5(b)$ ). The average distance moved per pulse $(50 \mathrm{~ms})$ for the applied pressure of $1.76 \mathrm{psi}, 2.84 \mathrm{psi}$ are $349 \mu \mathrm{m}$ and $919 \mu \mathrm{m}$ with corresponding velocities of $7.0 \mathrm{~mm} \mathrm{~s}^{-1}$ and $18.4 \mathrm{~mm} \mathrm{~s}^{-1}$, respectively. 

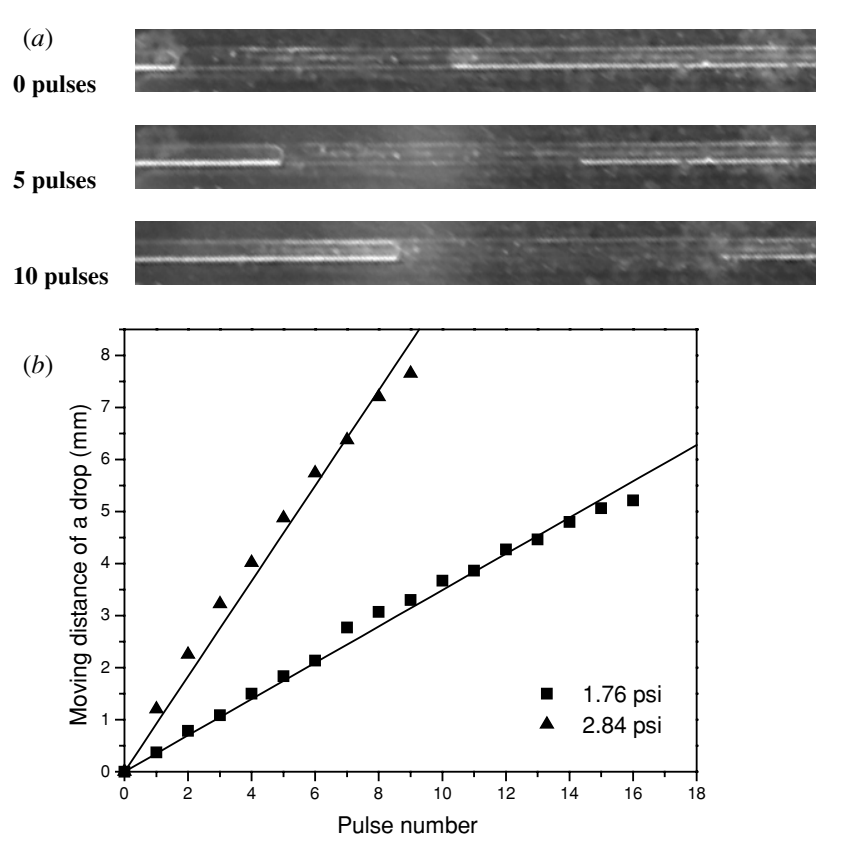

Figure 5. (a) Images showing pulsed drop motion in a COC microchannel using $50 \mathrm{~ms}$ pressure pulses at 1.76 psi. (b) Drop motion as a function of pulsed number at different pressures of $1.76 \mathrm{psi}(\boldsymbol{\square})$ and $2.84 \mathrm{psi}(\mathbf{\Lambda})$. The drop length is $5 \mathrm{~mm}$ and the channel dimension is $300 \mu \mathrm{m} \times 60 \mu \mathrm{m}$. The theoretical velocity curves (solid lines) were obtained using equation (2). Here $\Delta P=\Delta P_{\text {applied }}-\Delta P_{\text {hysteresis }}$ in this case. On the basis of the measured $\Delta P_{\text {hysteresis }}$, we have experimentally estimated the pressure head ( $\left.\Delta P_{\text {applied }}\right)$ for $1.76 \mathrm{psi}$ (50 ms pulse) to be about $0.09 \mathrm{psi}$ and for $2.84 \mathrm{psi}(50 \mathrm{~ms}$ pulse) to be about $0.11 \mathrm{psi}$.

In order to initialize drop motion, the applied pressure must overcome the pressure induced by contact angle hysteresis, $\Delta p_{\text {hysteresis }}$, and this pressure can be measured using a simple apparatus. A drop of water placed in a microchannel can be monitored while a pressure or vacuum is applied to one end of the channel. The difference in pressure (for our case, atmospheric pressure at one end and a slight vacuum at the other) at the moment the drop starts to move is $\Delta P_{\text {hysteresis }}$.
Table 1. Contact angle hysteresis in surface modified COC and glass devices.

\begin{tabular}{|c|c|c|c|}
\hline Substrate & lepth) & $\Delta P_{\text {hysteresis }}{ }^{\mathrm{a}}(\mathrm{Pa})$ & $\Delta \theta=\theta_{\mathrm{a}}-\theta_{\mathrm{r}}^{\mathrm{b}}$ \\
\hline & & & \\
\hline & & & \\
\hline \multicolumn{4}{|c|}{$\begin{array}{l}\text { a } \Delta P_{\text {hysteresis }}=(2 \sigma)\left(\frac{1}{w}+\frac{1}{d}\right)\left(\cos \theta_{\mathrm{r}}-\cos \theta_{\mathrm{a}}\right)= \\
\sigma\left(\frac{1}{w}+\frac{1}{d}\right) \sin \left(\frac{\Delta \theta}{2}\right) \sin \left(\theta_{\mathrm{s}}\right) \text { where } \Delta P_{\text {hysteresis }} \text { is the pressure } \\
\text { difference due to contact angle hysteresis, } \sigma \text { is the surface tension } \\
\left(72.75 \text { dynes } \mathrm{cm}^{-1} \text { for water }\right), w \text { is the channel width, } d \text { is the } \\
\text { channel depth. } \\
\text { b } \theta_{\mathrm{s}}, \theta_{\mathrm{a}}, \theta_{\mathrm{r}} \text { are static, advancing and receding contact angles, } \\
\Delta \theta=\theta_{\mathrm{a}}-\theta_{\mathrm{r}} \text { is the contact angle hysteresis, calculated using the } \\
\text { equation above from the static contact angle }\left(30^{\circ} \text { for glass and } 45^{\circ}\right. \\
\text { for treated COC). }\end{array}$} \\
\hline
\end{tabular}

The value for the contact angle hysteresis was then estimated from $\Delta P_{\text {hysteresis }}$, and the results are shown in table 1 . The contact angle hysteresis is around $19^{\circ}$ for COC and $23^{\circ}$ for glass devices respectively.

The contact angle hysteresis is also a function of velocity, and this variation can be calculated from the data shown in figure 4 . The velocity of a moving drop can be estimated by the Hagen-Poiseuille equation [29]:

$$
v=\frac{\left(\Delta P d^{2}\right)}{\mu L S}
$$

where $v$ is the mean velocity, $\mu$ is the viscosity, $L$ is the position of the moving meniscus and $S$ is a geometric constant. The geometric constants $S=60$ for COC and $S=50$ for glass are estimated on the basis of previous work [30]. $\Delta P$ is capillary pressure and can be calculated by Young-Laplace equation:

$$
\Delta P=(2 \sigma)\left(\frac{1}{w}+\frac{1}{d}\right) \cos \left(\theta_{\mathrm{s}}+\frac{\Delta \theta}{2}\right) .
$$

We can calculate $\Delta \theta$ as a function of velocity by combining equations (1) and (2). Figure 6 shows that the contact angle hysteresis is a weak function of velocity and rises gradually

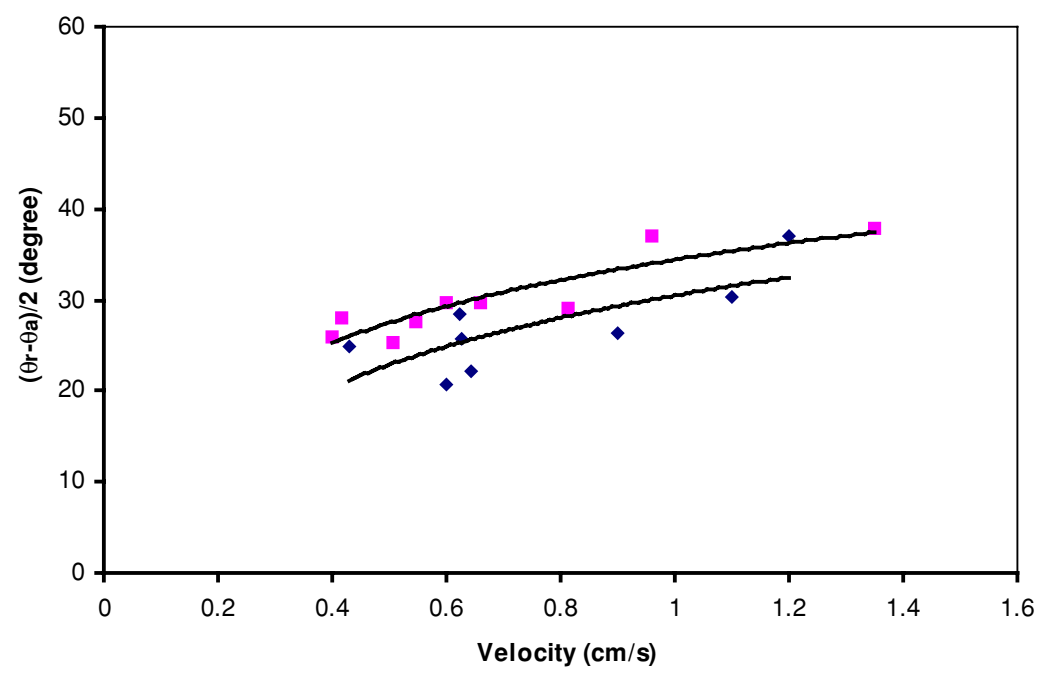

Figure 6. Estimated contact angle hysteresis $\left(\Delta \theta / 2=\left(\theta_{\mathrm{a}}-\theta_{\mathrm{r}}\right) / 2\right)$ as a function of velocity for COC $(\boldsymbol{\square})$ and glass $(\downarrow)$ devices. $\left(\theta_{\mathrm{s}}(\mathrm{glass})=\right.$ $30^{\circ}, \theta_{\mathrm{s}}(\mathrm{COC})=45^{\circ}$ in the calculation). 


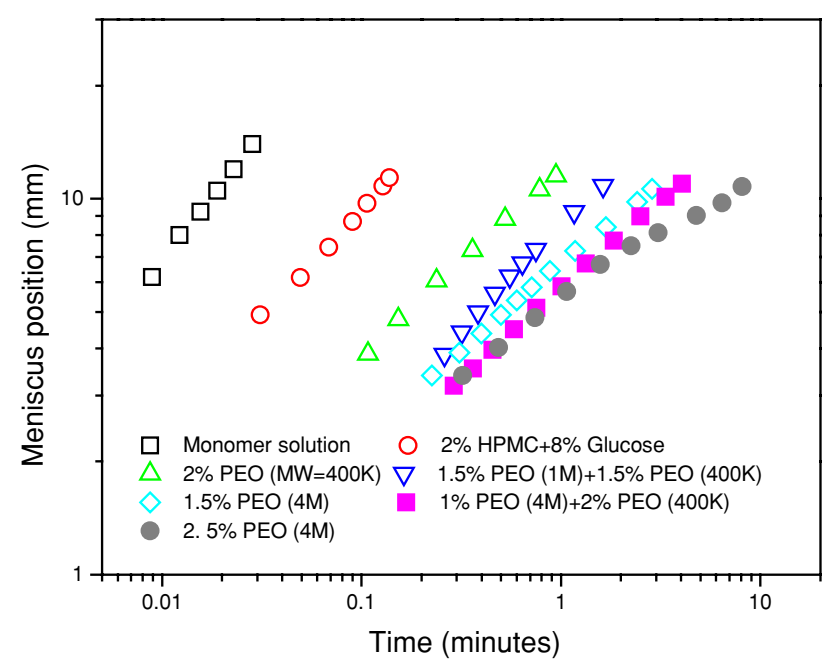

Figure 7. The meniscus position of sieving media inside the COC microchannel as a function of time.

as the velocity increases. This functionality is similar to that shown in other systems [31].

\section{Electrophoretic separation}

Surface modification facilitates many microfluidic operations. One example is loading sieving matrix for electrophoresis. We tested several commonly used separation media, including a crosslinked polyacrylamide monomer solution, various concentrations of PEO solution and HPMC solution. Note that PEO and HPMC are non-Newtonian shear thinning fluids, meaning that viscosity decreases as the shear rate increases.

Figure 7 shows the capillary flow behavior of these solutions. The velocity for the PEO solution in a range from less than $0.01 \mathrm{~mm} \mathrm{~s}^{-1}$ to $1 \mathrm{~mm} \mathrm{~s}^{-1}$ produces shear rates in the range of $0.3 \mathrm{~s}^{-1}$ to $30 \mathrm{~s}^{-1}$ for $60 \mu \mathrm{m}$ deep channels. The viscosity in this range of shear rate is of the order of several hundred cps. Those PEO solutions can be loaded into $1.5 \mathrm{~cm}$ long microchannel $(300 \mu \mathrm{m} \times 60 \mu \mathrm{m})$ solely by capillary force in approximately $10 \mathrm{~min}$. While high viscous media need extended periods of time to fill microchannels, others with low viscosity such as a polyacrylamide monomer solution (close to the viscosity of water $\sim 1 \mathrm{cp}$ ) and HPMC solution $\left(\sim 15 \mathrm{cp}\right.$ for shear rate $\left.>100 \mathrm{~s}^{-1}\right)$ can be easily loaded in a hydrophilic channel.

In order to test the separation ability of media in the COC devices, we performed electrophoresis of double-stranded DNA using a low viscosity HPMC sieving media with glucose as an additive [32]. Under a relatively low electric field $\left(\sim 40 \mathrm{~V} \mathrm{~cm}^{-1}\right)$, most of the fragments were well resolved with a resolution greater than 0.5 (figure 8). The theoretical plate number is calculated to be of the order of $10^{5} \mathrm{~m}^{-1}$. This number is relatively low most likely due to lacking control in injecting the sample plug. We have performed studies in which the resolution of the separated peaks can be greatly improved using electroloading onto a microfabricated electrode [33, 34].

\section{Conclusions}

In summary, we have investigated liquid handling in surface-modified polyolefin micofluidic devices. The

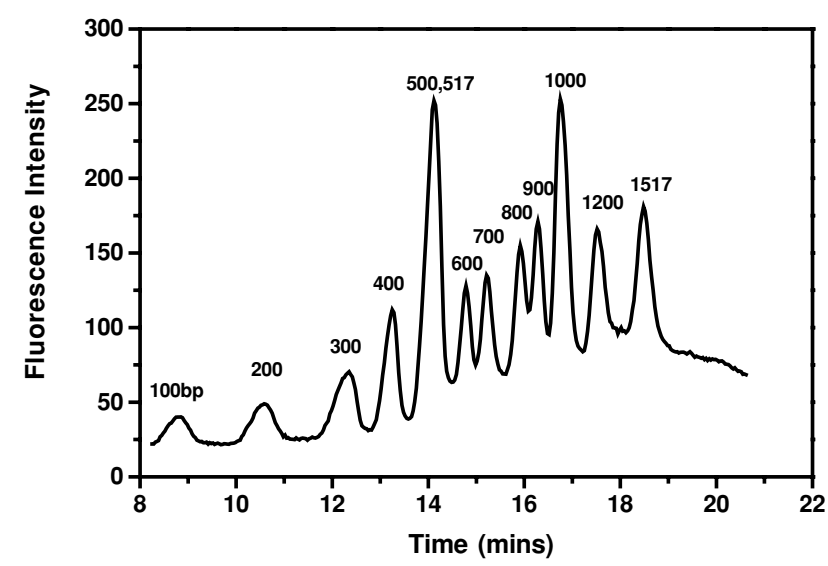

Figure 8. An electropherogram showing separation of YOYO-1 labeled double-stranded DNA in 2\% HPMC with $8 \%$ glucose additive. Intensity data were extracted directly from a video image sequence by averaging over a $20 \mu \mathrm{m}$ square detection area located $1.9 \mathrm{~cm}$ downstream from the injection cross $\left(E=39 \mathrm{~V} \mathrm{~cm}^{-1}\right)$.

surface was modified from hydrophobic to hydrophilic using a UV-mediated grafting technique. Capillary-driven flow, pulsed pumping and contact angle hysteresis were studied, and the separation of double-stranded DNA samples was demonstrated. Future work involving the selective pattern of hydrophilic/hydrophobic region [26] for metering and characteristics of electroosmosis flow in the surfacemodified devices would be beneficial for liquid handling and electrophoretic separations.

\section{Acknowledgments}

The authors acknowledge the support of the National Institutes of Health under the Grant NIHGRI P01-HG001984. The authors would also like to thank Zhishan Hua, Dr Onnop Srivannavit and Dr Xing Chen for their assistance in model fabrication and scanning electron microscope. The authors are grateful to Rohit Pal for constructive discussion and proofreading the manuscript.

\section{References}

[1] Paegel B M, Emrich C A, Wedemayer G J, Scherer J R and Mathies R A 2002 High-throughput DNA sequencing with a 96-lane capillary array electrophoresis bioprocessor Proc. Natl Acad. Sci. USA 99 574-9

[2] Emrich C A, Tian H, Medintz I and Mathies R A 2002 Microfabricated 384-lane capillary array electrophoresis bioanalyzer for ultra high-throughput genetic analysis Anal. Chem. 74 5076-83

[3] Kan C W, Fredlake C P, Doherty E A S and Barron A E 2004 DNA sequencing and genotyping in miniaturized electrophoresis systems Electrophoresis 25 3564-88

[4] Bousse L, Mouradian S, Minalla A, Yee H, William K and Dubrow R 2001 Protein sizing on a microchip Anal. Chem. 73 1207-12

[5] Herr A E and Singh A K 2004 Photopolymerized cross-linked polyacrylamide gels for on-chip protein sizing Anal. Chem. 76 4727-33

[6] Renzi R F, Stamps J, Horn B A, Ferko S, VanderNoot V A, West A A, Crocker R, Wiedenman B, Yee D and Fruetel J A 2005 Hand-held microanalytical instrument for chip-based 
electrophoretic separations of proteins Anal. Chem. 77 435-41

[7] Fruetel J A et al 2005 Microchip separations of protein biooxins using an integrated hand-held device Electrophoresis 26 1144-54

[8] Lagally E T, Scherer J R, Blazej R G, Toriello N M, Diep B A, Ramchandani M, Sensabaugh G F, Riley L W and Mathies R A 2004 Integrated portable genetic analysis microsystem for pathogen/infectious disease detection Anal. Chem. 76 3162-70

[9] Pal R et al 2005 An integrated microfluidic device for influenza and other genetic analyses device Lab Chip 5 1024-32

[10] Burns M A et al 1998 An Integrated nanoliter DNA analysis device Science 282 484-7

[11] Becker H and Gartner C 2000 Polymer microfabrication methods for microfluidic analytical applications Electrophoresis 21 12-26

[12] Fiorini G S and Chiu D T 2005 Disposable microfluidic devices: fabrication, function, and application Biotechniques 38 429-46

[13] McDonald J C and Whitesides G M 2002 Poly(dimethylsiloxane) as a material for fabricating microfluidic devices Acc. Chem. Res. 35 491-9

[14] Seong G H, Zhan W and Crooks R M 2002 Fabrication of microchambers defined by photopolymerized hydrogels and weirs within microfluidic systems: application to DNA hybridization Anal. Chem. 74 3372-7

[15] Moorthy J and Beebe D J 2003 In situ fabricated porous filters for microsystems Lab Chip 3 62-6

[16] Bohr T, Ogletree D F, Svec F and Frechet M J 2003 Surface functionalization of thermoplastic polymers for the fabrication of microfluidic devices by photoinitiated grafting Adv. Funct. Mater. 13 264-70

[17] Hu S, Ren X, Bachman M, Sims C E, Li G P and Allbritton N L 2003 Cross-linked coating for electrophoretic separation in poly(dimethylsiloxane) microchannels Electrophoresis 24 3679-88

[18] Hu S, Ren X, Bachman M, Sims C E, Li G P and Allbritton N L 2004 Surface-directed, graft polymerization within microfluidic channels Anal. Chem. 76 1865-70

[19] Data from Zeon Chemicals: http://www.zeonchemicals.com

[20] Hawkins K R and Yager P 2003 Nonlinear decrease of background fluorescence in polymer thin-films - a survey of materials and how they can complicate fluorescence detection in microTAS Lab Chip 3 248-52

[21] Koh C G, Tan W, Zhao M Q, Ricco A J and Fan Z H 2003 Integrating polymerase chain reaction, valving and electrophoresis in a plastic device for bacterials detection Anal. Chem. 75 4591-8

[22] Ahn C H, Choi J W, Beaucage G, Nevin J H, Lee J B, Puntambekar A and Lee J Y 2004 Disposable smart lab on a chip for point-of-care-clinical diagnostics Proc. IEEE 92 154-73

[23] Kameoka J, Craighead H G, Zhang H W and Henion J 2001 A polymeric microfluidic chip for $\mathrm{CE} / \mathrm{ME}$ determination of small molecules Anal. Chem. 73 1935-41

[24] Kameoka J, Orth R, Ilic B, Czaplewski D, Wachs T and Craighead H G 2002 An eledrospray ionization source for integration with microfluidics Anal. Chem. 74 5897-901

[25] Gaudioso J and Craighead H G 2002 Characterizing electroosmotic flow in microfluidic devices $J$. Chromatogr. A 971 249-53

[26] Li C, Yang Y, Craighead H G and Lee K H 2005 Isoelectric focusing in cylic olefin copolymer microfluidic channels coated by polyacrylamide using a UV photografting method Electrophoresis 26 1800-6

[27] Duffy D C, McDonald J C, Schueller O J A and Whiteside G M 1998 Rapid prototyping of microfluidic systems in poly(dimethylsiloxane) Anal. Chem. 70 4974-84

[28] Handique K, Burke D T, Mastrangelo C H and Burns M A 2000 Nanoliter liquid metering in microchannels using hydrophobic patterns Anal. Chem. 72 4100-9

[29] Bird R B, Stewart W E and Lightfoot E N 1994 Transport Phenomena 2nd edn (New York: Wiley)

[30] Sammarco T S and Burns M A 1999 Thermocapillary pumping of discrete drops in microfabricated analysis devices AIChE J. 45 350-66

[31] Seeberg J E and Berg J C 1992 Dynamic wetting in the low capillary number regime Chem. Eng. Sci. 47 4455-64

[32] Xu F, Jabasin M and Baba Y 2002 DNA separation by microchip electrophoresis using low-viscosity hydroxypropylmethylcellulose-50 solutions enhanced by polyhydroxy compounds Electrophoresis 23 3608-14

[33] Brahmasandra S N, Ugaz V M, Burke D T, Mastrangelo C H and Burns M A 2001 Electrophoresis in microfabricated devices using photopolymerized polyacrylamide gels and electrode-defined sample injection Electrophoresis 22 300-11

[34] Ugaz V M, Brahmasandra S N, Burke D T and Burns M A 2002 Cross-linked polyacrylamide gel electrophoresis of single-stranded DNA for microfabricated genomic analysis systems Electrophoresis 23 1450-9 